\title{
ÜBER DEN ERFOLG EINER HOCHLAGENAUFFORSTUNG AUF VERSCHIEDENEN STANDORTSTYPEN (STILLBERG IM DISCHMATAL, DAVOS)
}

\author{
W. SCHÖNENBERGER
}

\section{Abstract}

In a NE-exposed avalanche area at the alpine timberline survival percentage and height growth rate of a 12 years old test planting with Larch, Spruce and Mountain Pine was examined on the most important site types (Fig. 1). The correlation between height growth of Larch and Spruce and the climatic factors irradiation, wind speed and date of snow melting is shown in a multivariate model (Fig. 2).

In der Versuchsfläche Stillberg wurden im Zusammenhang mit den standortskundlichen Untersuchungen (vgl. BLASER 1975) Test-Aufforstungen angelegt mit dem Ziel, die Ursachen von Erfolg und Misserfolg auf den verschiedenen Standorten kennenzulernen und der forstlichen Praxis die Grundlagen für eine möglichst erfolgversprechende Aufforstungsmethode in Hochlagen zu bieten.

Erfolg oder Misserfolg einer Aufforstung dokumentieren sich vorerst einmal im Prozentsatz der Bäume, welche die Anfangsschwierigkeiten überstanden haben und einen Beitrag an die Bestandesbildung, das Ziel jeder Aufforstung, leisten können. Bestandesbildung ist unter den extremen Bedingungen der Waldgrenz- und Kampfzone, in welcher die Versuchsfläche Stillberg liegt, nur an jenen Standorten möglich, welche neben einer genügenden Überlebensrate auch einen nennenswerten Höhenzuwachs erlauben.

Das Resultat einer zwölfjährigen Pflanzung soll ein Bild geben von der Grössenordnung der Erfolgsaussichten. In dieser Pflanzung wurden je 16 Lärchen und Fichten sowie 8 Bergföhren aus Hochlagenprovenienzen bei einem Pflanzabstand von $50 \mathrm{~cm}$ zwischen den Einzelbäumen in Einheitsflächen mit Wiederholungen auf die typischen Standorte im Versuchsgelände verteilt.

\begin{tabular}{lccccc} 
& Anzahl & Alter & \multicolumn{3}{c}{ Pflanzungszustand nach 12 Jahren } \\
& Gepflanzte & Jahre & Überlebens-\% & Mittl. Höhe cm & Streuung \\
Lärche & 688 & 2 & 69,5 & 45,9 & 23,9 \\
Fichte & 688 & 3 & 60,3 & 27,0 & 11,0 \\
Bergföhre & 344 & 2 & 48,8 & 29,0 & 14,3
\end{tabular}

Die Stammhöhe ist im Hinblick auf Bestandesbildung und Wechselwirkung mit der Schneedecke ein geeignetes Zuwachs-Mass, darf aber nicht als Mass für die Stoffproduktion betrachtet werden.

Ausfälle bis zu 50\% und der sehr geringe Höhenzuwachs zeigen die grosse Gefährdung der Aufforstungen in solch extremen Lagen. Die Streuung ist bei dieser summarischen Zusammenfassung aller Standorte natürlich sehr gross, meist etwa die 
Hälfte des Mittelwertes. Sie beruht wohl in erster Linie auf den Standortsunterschieden.

Für die Darstellung des Aufforstungserfolges auf den verschiedenen Standortstypen diene die von AULITZKY (1963) eingeführte Ökogramm-Form. Von den pflanzensoziologischen, mikroklimatischen und schneekundlichen Verhältnissen eines repräsentativen Geländequerschnittes auf Stillberg ausgehend zeigt das Ökogramm (Abb. 1) die Verteilung der Überlebensprozente und der Stammhöhen der erwähnten 12-jährigen Pflanzung. Daraus ist ersichtlich, dass nicht alle Baumarten gleich empfindlich auf die Standortsunterschiede reagieren. Die Variation der Stammhöhe ist bei der Lärche weitaus grösser als bei der Fichte, wo der Zuwachs über alle Standorte ziemlich ausgeglichen ist und die Überlebensrate innerhalb engerer Grenzen schwankt. Dies mag z.T. daher rühren, dass die Fichte in dieser Höhenlage selbst an den besten Standorten die Grenze der Lebensmöglichkeit erreicht.

Erfolgversprechend sind auf Stillberg laut Okogramm in erster Linie die E-exponierten Einhänge mit Junipero-Arctostaphyletum, wo die Überlebens- und Zuwachsrate gleichzeitig recht hoch ist. Dieser Standort ist charakterisiert durch hohe Einstrahlung, geringe Windgeschwindigkeit und mittlere Schneebedeckungsdauer. Es sind somit weder Frosttrocknis- noch Pilzschäden von Bedeutung zu erwarten. Dank günstigen sommerlichen Verhältnissen sind hier die Bäume recht vital und resistent gegen winterliche Schädigungen. Besonders die Lärche vermag die Gunst dieses Standorts auszunützen.

Ohne künstliche Hilfsmassnahmen schwer aufzuforsten sind dagegen die N-Einhänge mit Empetro-Vaccinietum mit den relativ hohen Ausfällen und dem sehr geringen Zuwachs. An diesem kühlen und schattigen Standort mit der kurzen Vegetationsperiode bleibt den Bäumchen kaum Zeit, genügend Reserven anzulegen und das Wachstum rechtzeitig abzuschliessen, was die Anfälligkeit für Schädigungen erhöht. Hier sind neben dem ungünstigen Mikroklima in hohem Masse Pilzkrankheiten für den Misserfolg verantwortlich.

In Runsen- und Rippenlagen ergeben sich Diskrepanzen zwischen Überlebensund Zuwachsrate. In den Runsen mit dem konkurrenzkräftigen Calamagrostietum villosae werden die meisten Bäumchen schon frühzeitig durch Lichtmangel erstickt, den überlebenden bleibt nur Raum in die Höhe. Die Lawinenniedergänge, welche in diesen Runsen kanalisiert werden, verursachen vorläufig noch kaum mechanische Schäden, gewinnen aber mit Sicherheit in einem späteren Zeitpunkt, wenn die Stämmchen dicker und weniger elastisch werden, grösste Bedeutung.

Auf den Geländerippen, in der niedrigen und offenen Gesellschaft des CetrarioLoiseleurietums, herrschen geradezu gegenteilige Bedingungen. Der Prozentsatz der Überlebenden ist relativ hoch, aber die Bäumchen haben an diesen windexponierten und schneearmen Standorten Mühe, über die schützende Vegetationsdecke hinauszuwachsen. Wiederholt fallen die herausragenden Teile im Winter der Frosttrocknis zum Opfer, oder die ungeschützten frischen Triebe erfrieren im Frühjahr. Der Habitus solcher Pflanzen gleicht nach einigen Jahren eher einem Busch als einem Baum.

Flachere Geländepartien im unteren Teil der Versuchsfläche werden vom $R$ hododendro-Vaccinietum besiedelt. Dank der tieferen Lage ist das Klima etwas milder. Dieser Vorteil wird aber teilweise aufgehoben durch die extrem hohe und lange Schneebedeckung.

Im Unterschied zum Ökogramm von AULITZKY (1963), in welchem die Erfolgschancen einer Aufforstung nach der Dichte des natürlichen Jungwuchses von Arve 


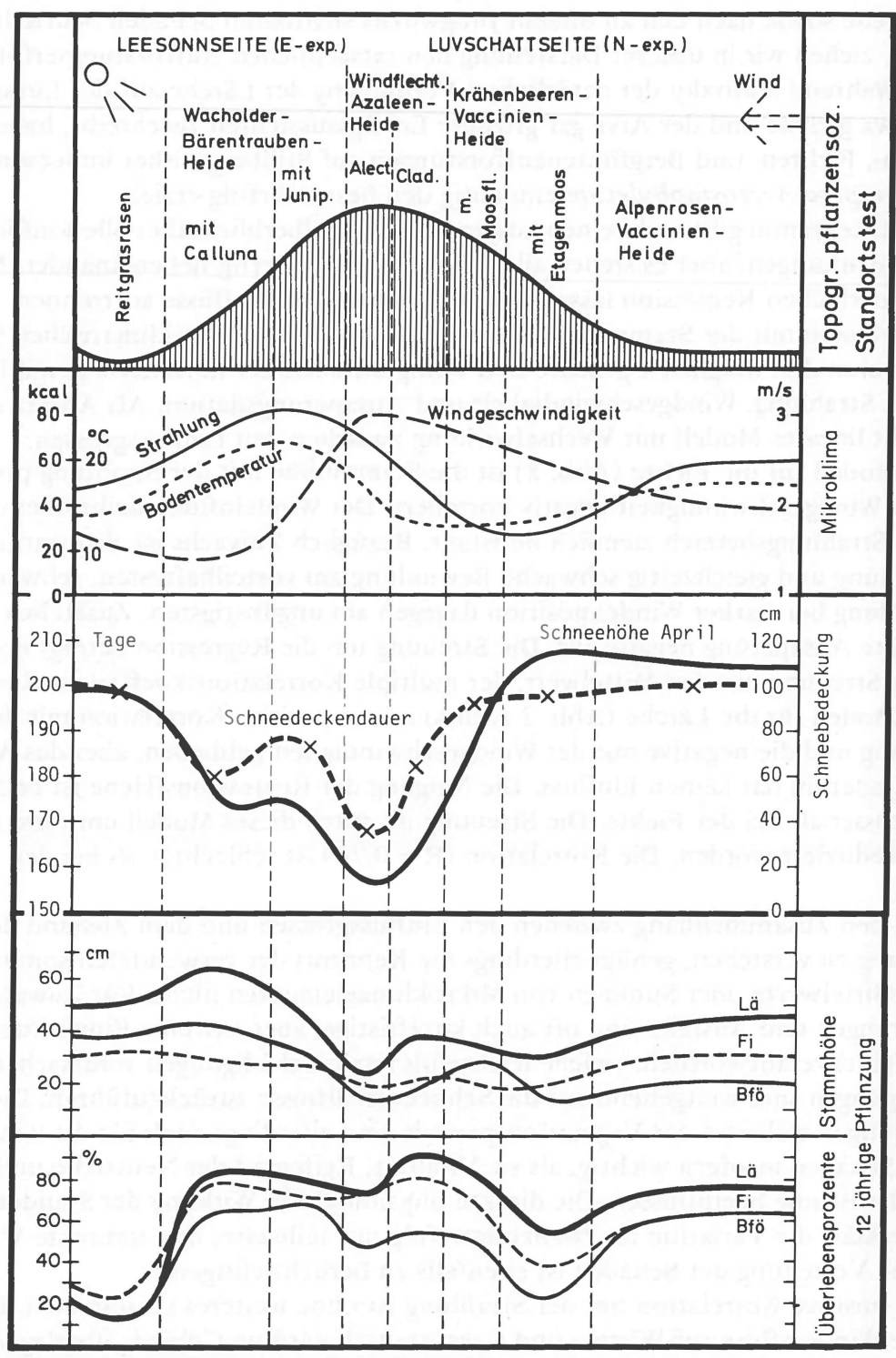

$A b b$. 1. Ökographische Übersicht über einen schematischen Geländequerschnitt in der NE-exponierten Versuchsfläche Stillberg (2000-2230 m ü.M.) nach TURNER, ROCHAT \& STREULE (1975). Pflanzengesellschaften nach KUOCH (1970); globale Hangbestrahlung in $\mathrm{kcal} / \mathrm{cm}^{2}$ summiert über eine mittlere Vegetationsperiode vom 15.5. bis 30.9., TURNER (1966); mittlere Geschwindigkeit des Tagwindes in $\mathrm{m} / \mathrm{s}$ in $1 \mathrm{~m}$ über der Bodenoberfläche während der Vegetationsperiode, NÄGELI (1971); Mittelwerte der Bodentemperatur in $8 \mathrm{~cm}$ Tiefe während der Vegetationsperiode, TURNER et al. (1975); mittlere April-Schneehöhe 19711974; Dauer der regulären Schneebedeckung, 11-jährige Mittelwerte 1959/60-1969/70, IN DER GAND \& RYCHETNIK (1970); Stammhöhe und Überlebensprozente von Lärche, Fichte und aufrechter Bergföhre nach SCHÖNENBERGER (1975). 
und Lärche sowie nach den an diesem Jungwuchs sichtbaren Schäden beurteilt wurden, ziehen wir in unserer Darstellung den tatsächlichen Aufforstungserfolg heran. Während Aulitzky der natürlichen Verjüngung der Lärche auf der Luvschattseite etwa gleiche und der Arve gar grössere Erfolgsaussichten zuschreibt, haben die Lärchen-, Fichten- und Bergföhrenaufforstungen auf Stillberg bisher im leesonnseitigen Junipero-Arctostaphyletum eindeutig den besten Erfolg erzielt.

Das Ökogramm gibt wohl einen ausgezeichneten Überblick über alle Einflüsse und Auswirkungen, aber es stehen alle Faktoren gleichwertig nebeneinander. Mittels einer mehrfachen Regression lassen sich die wichtigsten Einflüsse auftrennen. Für die Regression mit der Stammhöhe wurden aus der Vielzahl der klimatischen Standortsfaktoren drei möglichst primäre und wenig miteinander korrelierte gewählt, nämlich Strahlung, Windgeschwindigkeit und Ausaperungsdatum. Als Ansatz diente ein nicht lineares Modell mit Wechselwirkung zwischen den Einflussgrössen.

Im Modell für die Fichte (Abb. 2) ist die Stammhöhe mit der Strahlung positiv, mit der Windgeschwindigkeit negativ korreliert. Der Windeinfluss bleibt über den ganzen Strahlungsbereich ziemlich konstant. Bezüglich Zuwachs ist also starke Bestrahlung und gleichzeitig schwache Bewindung am vorteilhaftesten, schwache Bestrahlung bei starker Windexposition dagegen am ungünstigsten. Zusätzlich wirkt sich späte Ausaperung negativ aus. Die Streuung um die Regression beträgt noch $1 / 3$ der Streuung um den Mittelwert, der multiple Korrelationskoeffizient $R=0,86$.

Im Modell für die Lärche (Abb. 2 rechts) ist die positive Korrelation mit der Strahlung und die negative mit der Windgeschwindigkeit geblieben, aber das Ausaperungsdatum hat keinen Einfluss. Die Neigung der Regressionsfläche ist bedeutend grösser als bei der Fichte. Die Streuung ist durch dieses Modell um rund die Hälfte reduziert worden. Die Korrelation $(R=0,74)$ ist schlechter als bei der Fichte.

Um den Zusammenhang zwischen den Einflussgrössen und dem Zustand der Pflanzung zu verstehen, genügt allerdings die Kenntnis der verwendeten sommerlichen Mittelwerte oder Summen von Mikroklimaelementen nicht. Für Zuwachsminderungen und Ausfälle sind oft auch kurzfristige, aber extreme Einwirkungen (z.B. Frost) verantwortlich, welche leichte bis letale Schädigungen verursachen. Schädigungen sind weitgehend auf die Schneeverhältnisse zurückzuführen. Die Bedingungen während der Vegetationsperiode sind allerdings auch für die winterlichen Schäden insofern wichtig, als sie Vitalität, Reifegrad der Neutriebe und Resistenz der Bäume beeinflussen. Die direkte physiologische Wirkung der Standortsfaktoren erklärt die Variation im Pflanzungserfolg nur teilweise; ihre indirekte Wirkung über die Verteilung der Schäden ist ebenfalls zu berücksichtigen.

Die positive Korrelation mit der Strahlung ist ohne weiteres verständlich. Der direkte Windeinfluss auf Wärme- und Gasaustausch wird im Gelände überlagert durch seine hervorragende Bedeutung für die Verteilung der Schneedecke, welche wiederum zusammen mit der Lawinentätigkeit und der Strahlung die Schneedeckendauer bzw. die Dauer der Vegetationsperiode mitbestimmt. Dadurch kommen die winterlichen Verhältnisse mit ihren oft unberechenbaren Folgen ins Spiel (Frosttrocknis bei Schneemangel, Pilzschäden bei Schnee-,,Schutz"). Dass das Ausaperungsdatum auf den Lärchenzuwachs keinen Einfluss hat, dürfte in der Tatsache begründet sein, dass die Lärche dank des Nadelverlustes im Winter weniger anfällig für Pilzinfektionen ist.

Es sei auch darauf hingewesen, dass sich in einer jüngeren Phase der Aufforstung 
und bei differenzierterer Klassierung der Einflussgrössen der Windschutz an Stellen mit sehr geringer Einstrahlung (ca. $35 \mathrm{kcal} / \mathrm{cm}^{2} /$ Veg.per.) negativ auswirkte (TURNER 1971).

\section{FICHTE}
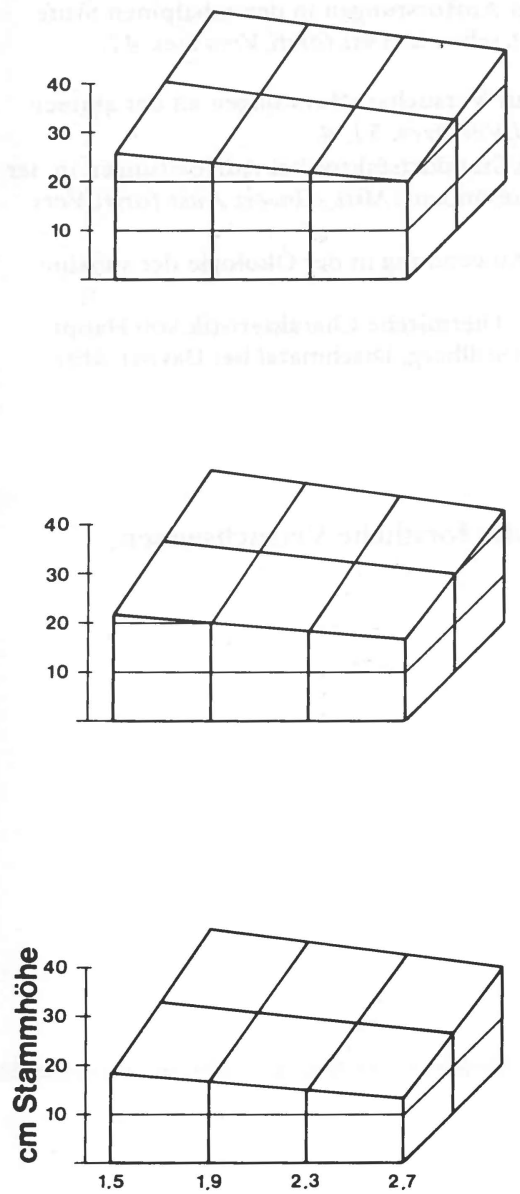

$\mathrm{m} / \mathrm{s}$ Windgeschwindigkeit

\section{LÄRCHE}

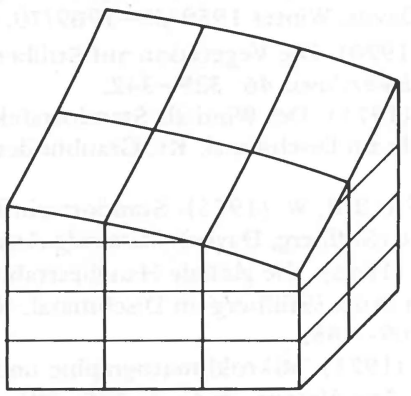

28. April

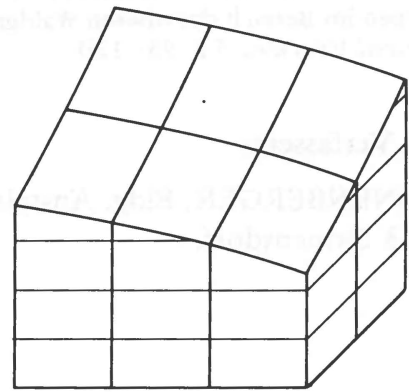

13. Mai

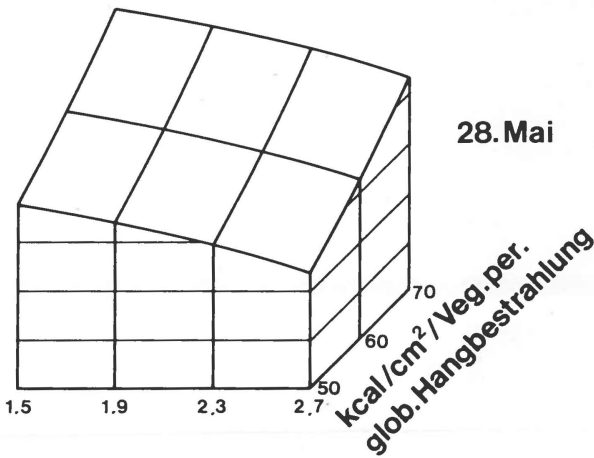

$A b b$. 2. Regressionsmodell für die Stammhöhe einer 12-jährigen Pflanzung in Abhängigkeit von globaler Hangbestrahlung, Windgeschwindigkeit und Ausaperungsdatum unter Geländebedingungen, Versuchsfläche Stillberg. 


\section{LITERATUR}

AULITZKY, H. (1963): Grundlagen und Anwendung des vorläufigen Wind-Schnee-Ökogrammes. Mitt.forstl.Bundes-Vers'anst.Mariabrunn 60: 763-834.

BLASER, P. (1975): Standortsfaktoren und Bodenbildung in einem subalpinen Lawinenanrissgebiet (Stillberg bei Davos). Verh. Ges. Ökol. Wien 1975: 49-54.

BLASER, P. (in Vorbereitung): Der Boden als Standortsfaktor bei Aufforstungen in der subalpinen Stufe (Stillberg im Dischmatal, Kt. Graubünden). Mitt.eidg.Anst.forstl.Vers'wes.

IN DER GAND, H., \& RYCHETNIK, J. (1970): Dauer der regulären Schneebedeckung der Pegelstandorte im Bereich der Testpflanzungen EAFV 1959/1961/1962, Versuchsfläche Stillberg/Davos, Winter 1959/60-1969/70. Interner Ber. 507, EISLF Weissfluhjoch-Davos.

KUOCH, R. (1970): Die Vegetation auf Stillberg (Dischmatal, Kt. Graubünden). Mitt.schweiz. Anst.forstl.Vers'wes. 46: 329-342.

NÄGELI, W. (1971): Der Wind als Standortsfaktor bei Aufforstungen in der subalpinen Stufe (Stillbergalp im Dischmatal, Kt. Graubünden). Mitt.schweiz.Anst.forstl.Vers'wes. 47: 33-147.

SCHÖNENBERGER, W. (1975): Standortseinflüsse auf Versuchsaufforstungen an der alpinen Waldgrenze (Stillberg, Davos). Mitt.eidg.Anst.forstl.Vers'wes. 51, 4.

TURNER, H. (1966): Die globale Hangbestrahlung als Standortsfaktor bei Aufforstungen in der subalpinen Stufe (Stillberg im Dischmatal, Kt. Graubünden). Mitt.scbweiz.Anst.forstl.Vers' wes. 42: 109-168.

TURNER, H. (1971): Mikroklimatographie und ihre Anwendung in der Ökologie der subalpinen Stufe. Ann.Meteor., N.F., 5: 275-281.

TURNER, H., ROCHAT, P., \& STREULE, A. (1975): Thermische Charakteristik von Hauptstandortstypen im Bereich der oberen Waldgrenze (Stillberg, Dischmatal bei Davos). Mitt. eidg.Anst.forstl.Vers'wes. 51: 95-120.

Anschrift des Verfassers:

W. SCHÖNENBERGER; Eidg. Anstalt für das forstliche Versuchswesen; $\mathrm{CH}-8903$ Birmensdorf. 